\title{
Deux améliorations concurrentes des PID
}

\section{Two competing improvements of PID controllers: A comparison}

\author{
Michel Fliess ${ }^{1,3}$ et Cédric Join $2,3,4$
}

${ }^{1}$ LIX (CNRS, UMR 7161),École polytechnique, 91128 Palaiseau, France, Michel.Fliess@polytechnique.edu

${ }^{2}$ CRAN (CNRS, UMR 7039), Université de Lorraine, BP 239, 54506 Vandœuvre-lès-Nancy, France,

Cedric.Join@univ-lorraine.fr

${ }^{3}$ AL.I.E.N. (ALgèbre pour Identification \& Estimation Numériques), 7 rue Maurice Barrès, 54330 Vézelise, France,

\{michel.fliess, cedric.join\}@alien-sas.com

${ }^{4}$ Projet Non-A, INRIA Lille - Nord-Europe, France

RÉSUMÉ. Aujourd'hui, «commande sans modèle », ou «MFC », et «commande par rejet actif de perturbations », ou "ADRC », sont les approches les plus en vue pour préserver les avantages des PID, si populaires dans l'industrie, tout en atténuant leurs carences. Après un bref rappel sur MFC et ADRC, plusieurs exemples démontrent la supériorité du sans-modèle car permettant d'embrasser une classe beaucoup plus vaste de systèmes.

ABSTRACT. In today's literature Model-Free Control, or MFC, and Active Disturbance Rejection Control, or ADRC, are the most prominent approaches in order to keep the benefits of PID controllers, that are so popular in the industrial world, and in the same time for attenuating their severe shortcomings. After a brief review of MFC and ADRC, several examples show the superiority of MFC, which permits to tackle most easily a much wider class of systems.

MOTS-CLÉS. PID, commande sans modèle, commande par rejet actif des perturbations, commande par platitude.

KEYWORDS. PID, model-free control, MFC, active disturbance rejection control, ADRC, flatness-based control.

\section{Introduction}

On connaît la domination, écrasante dans l'industrie, des correcteurs de type «proportionnel-intégraldérivé », ou «PID » (voir, par exemple, [Åström \& Hägglund (2006)], [Åström \& Murray (2008)], [O'Dwyer (2009)]). À côté d'avantages aussi considérables que reconnus, singulièrement la simplicité conceptuelle et l'inutilité d'une modélisation mathématique, des manques flagrants conduisant trop souvent à une mise en œuvre pénible et à des performances médiocres. La recherche universitaire, si active en automatique, dite parfois «moderne » depuis plus de cinquante ans, y trouve en bonne part sa motivation. Elle suppose fréquemment une description par équations différentielles ou aux différences. Ses contributions, théoriques et pratiques, sont incontestables (voir, par exemple, [Åström \& Murray (2008)], [Åström \& Kumar (2014)]). Afin de ne pas trop alourdir cette publication, contentons-nous, ici, de citer deux apports, aux applications aussi nombreuses que diverses : le filtre de Kalman [Kalman (1960), Kalman \& Bucy (1961)] et la platitude [Fliess et coll. (1995), Fliess et coll. (1999)].

Durant toutes ces années, on a tenté à maintes reprises, avec, semble-t-il, un succès mitigé jusqu'à récemment, de garder les avantages des PID tout en gommant leurs défauts. Deux voies, plutôt nouvelles, prévalent à l'heure actuelle :

1. la «commande sans modèle » [Fliess \& Join (2013)], ou model-free control, désignée ici par MFC, son acronyme anglais ;

2. la «comande par rejet actif de perturbations », ou active disturbance rejection control, désignée 
aussi par son acronyme anglais, $A D R C$. Cette méthode, issue avant tout des travaux de [Han (2009)] en Chine, a été développée par divers auteurs. Voir, par exemple,

- les articles signés par [Aguilar-Ibañez et coll. (2017)], [Feng \& Guo (2017)], [Gao (2014)] ${ }^{1}$, [Guo \& Zhou (2015)], [Huang \& Xue (2014)], [Inoue \& Ishida (2016)], [Qi et coll. (2017)], [Madoński \& Herman (2015)], [Polóni et coll. (2018)], [Tavasoli \& Enjilela (2017)], [Vincent et coll. (2011)], [Wu \& coll. (2015)], [Yao \& Deng (2017)], [Zhang et coll. (2014)], [Zheng \& Gao (2016)]);

- les livres de [Guo \& Zhao (2016)] et [Sira-Ramírez et coll. (2017)].

MFC et ADRC ont permis bien des applications, parfois spectaculaires. Les bibliographies des références plus haut sur l'ADRC en fournissent une liste assez complète. Pour le MFC, renvoyons à la bibliographie de [Fliess \& Join (2013)], à [Abouaïssa et coll. (2017a), Abouaïssa et coll. (2017b)] et à leurs références.

Des adeptes de l'ADRC ont publié des critiques, aussi vives qu'infondées, contre le MFC (voir, par exemple, [Madoński \& Herman (2012)]). L'ignorance totale chez d'autres, comme [Guo \& Zhao (2016)], des techniques du MFC aboutit à un exposé partial. Certains, comme [Cortés-Romero et coll. (2017)], effacent l'écart béant les séparant. Cet article use d'un droit de réponse légitime. Il vise à rétablir les faits, en exploitant, après avoir résumé les deux approches, plusieurs exemples académiques ${ }^{2}$. Puisse une discussion ouverte et fructueuse s'ensuivre ${ }^{3}$.

Le paragraphe 2 résume MFC et ADRC. On s'y inspire pour l'ADRC du nouveau livre [Sira-Ramírez et coll. (2017)], plus clair. Le suivant exhibe des simulations numériques, qui vont du linéaire au non-linéaire en terminant par une équation aux dérivées partielles. Ils tendent à prouver la supériorité du sans-modèle. La conclusion évoque aussi :

1. l'influence sur la compréhension de questions naturelles;

2. les retards ;

3. les équations aux dérivées partielles.

\section{Les deux approches}

\section{1. $M F C$}

\section{Emploi d'un théorème d'approximation}

On se restreint, afin de simplifier l'écriture, à un système $S I S O^{4}$, c'est-à-dire avec une seule commande $u$ et une seule sortie $y$. On suppose la «fonctionnelle » correspondant à $y$, c'est-à-dire la fonction de la

1. Certains auteurs, comme [Gao (2014)], ont voulu faire remonter quelques-uns des principes de l'ADRC plus haut dans le temps, à [Poncelet (1870)] en particulier.

2. Aux lecteurs de comparer les applications concrètes grâce aux références bibliographiques. Une telle évaluation est, dans le cadre de cet article, impossible.

3. L'importance pour l'ADRC de la contribution chinoise est décisive. On doit souligner d'autant plus la grande qualité de maints travaux en Chine sur le MFC (voir, par exemple, [Ticherfatine \& Quidam (2017), Wang et coll. (2017), Wang et coll. (2016), Zhang et coll. (2018)]).

4. Acronyme de Single-Input Single-Output. 
fonction $u$, causale, ou non-anticipative :

$$
\forall t>0, \quad y(t)=\mathcal{F}(u(\tau) \mid 0 \leqslant \tau \leqslant t)
$$

\section{$\mathcal{F}$ dépend}

— du présent et du passé, mais pas du futur;

- de perturbations variées ;

- de conditions initiales en $t=0$.

Exemple. Populaires en automatique (voir, par exemple, [Lamnabhi-Lagarrigue (1994), Rugh (1981)]) et dans divers domaines des sciences appliquées, les « séries de Volterra» sont des fonctionnelles du type

$$
\begin{aligned}
y(t)= & h_{0}(t)+\int_{0}^{t} h_{1}(t, \tau) u(\tau) d \tau+ \\
& \int_{0}^{t} \int_{0}^{t} h_{2}\left(t, \tau_{2}, \tau_{1}\right) u\left(\tau_{2}\right) u\left(\tau_{1}\right) d \tau_{2} d \tau_{1}+\ldots \\
& \int_{0}^{t} \ldots \int_{0}^{t} h_{\nu}\left(t, \tau_{\nu}, \ldots, \tau_{1}\right) u\left(\tau_{\nu}\right) \ldots u\left(\tau_{1}\right) d \tau_{\nu} \ldots d \tau_{1} \\
& +\ldots
\end{aligned}
$$

Elles apparaissent, notamment, comme solutions d'équations différentielles ordinaires assez générales (voir, par exemple, [Lamnabhi-Lagarrigue (1994)], [Rugh (1981)] et [Fliess et coll. (1983)]).

On introduit

— un compact $\mathcal{I} \subset[0,+\infty[$;

— un compact $\mathcal{C} \subset C^{0}(\mathcal{I})$, où $C^{0}(\mathcal{I})$ est l'ensemble des fonctions continues $\mathcal{I} \rightarrow \mathbb{R}$, muni de la topologie de la convergence uniforme.

Soit $\mathfrak{S}$ la $\mathbb{R}$-algèbre de Banach des fonctionnelles (1), causales et continues, $\mathcal{I} \times \mathcal{C} \rightarrow \mathbb{R}$. D'après le théorème de Stone-Weierstraß (voir, par exemple, [Choquet (2000), Rudin (1967)]), toute sous-algèbre, contenant une constante non nulle et séparant les éléments de $\mathcal{I} \times \mathcal{C}$, est dense dans $\mathfrak{S}$. Soit $\mathfrak{A} \subset \mathfrak{S}$ l'ensemble des fonctionnelles satisfaisant des équations différentielles algébriques du type

$$
E\left(y, \dot{y}, \ldots, y^{(a)}, u, \dot{u}, \ldots, u^{(b)}\right)=0
$$

où $E$ est un polynôme à coefficients réels. Selon [Fliess \& Join (2013)], $\mathfrak{A}$ est dense dans $\mathfrak{S}$. Il est ainsi loisible de supposer que le système considéré est «bien » approché par un système (2). Soit un entier $\nu$, $1 \leqslant \nu \leqslant a$, tel que

$$
\frac{\partial E}{\partial y^{(\nu)}} \not \equiv 0
$$

D'où, localement, d'après le théorème des fonctions implicites,

$$
y^{(\nu)}=\mathcal{E}\left(y, \dot{y}, \ldots, y^{(\nu-1)}, y^{(\nu+1)}, \ldots, y^{(a)}, u, \dot{u}, \ldots, u^{(b)}\right)
$$

Il en découle le système « ulta-local »

$$
y^{(\nu)}=F+\alpha u
$$

Souvent, en pratique, on sélectionne $\nu=1$. Voir [Fliess \& Join (2013)] pour une explication. On rencontre parfois $\nu=2$. Jamais plus. Le praticien choisit $\alpha \in \mathbb{R}$ tel que les trois termes de (3) aient le même ordre de grandeur. Il en découle qu'une identification précise du paramètre $\alpha$ est sans objet. 
Reprenons (3) avec $\nu=2$. Un «PID intelligent », ou «iPID », est défini par

$$
u=-\frac{F_{\text {est }}-\ddot{y}^{*}-K_{P} e-K_{I} \int e-K_{D} \dot{e}}{\alpha}
$$

où

$-y^{*}$ est la trajectoire de référence;

$-e=y-y^{*}$ est l'erreur de poursuite ;

- $K_{P}, K_{I}, K_{D} \in \mathbb{R}$ sont les gains;

- $F_{\text {est }}$ est une estimée de $F$.

Il vient

$$
\ddot{e}=K_{P} e+K_{I} \int e+K_{D} \dot{e}+F-F_{\text {est }}
$$

Si l'estimée $F_{\text {est }}$ est «bonne », c'est-à-dire $F-F_{\text {est }} \bumpeq 0$, déterminer les gains est immédiat pour obtenir une «bonne » poursuite. C'est un avantage important par rapport aux PID classiques.

Si $K_{D}=0$, on a un «PI intelligent , ou «iPI »,

$$
u=-\frac{F_{\text {est }}-\ddot{y}^{*}-K_{P} e-K_{I} \int e}{\alpha}
$$

Si $K_{I}=0$, on a un «PD intelligent», ou «iPD»,

$$
u=-\frac{F_{\text {est }}-\ddot{y}^{*}-K_{P} e-K_{D} \dot{e}}{\alpha}
$$

Avec $\nu=1$, on aboutit à un «P intelligent $», \mathrm{ou} \ll \mathrm{iP} »$,

$$
u=-\frac{F_{\mathrm{est}}-\ddot{y}^{*}-K_{P} e}{\alpha}
$$

C'est le correcteur le plus courant dans la pratique du $\mathrm{MFC}^{5}$. La dynamique (5) devient

$$
\dot{e}=K_{P} e+F-F_{\text {est }}
$$

Remarque 1. On démontre [d'Andréa-Novel et coll. (2010), Fliess \& Join (2013)] qu'un iP (resp. iPD) est équivalent, en un certain sens, à un PI (resp. PID) classique. On comprend ainsi, sans doute pour la première fois,

- l'universalité industrielle des PID,

- la difficulté de leur réglage.

Remarque 2. Dans tous les exemples $\mathrm{MIMO}^{6}$ concrets rencontrés jusqu'à présent, on a toujours pu se ramener à des systèmes de type (3) en parallèle ou en cascade. La mise en place des correcteurs intelligents reste donc facile (voir [Bekcheva et coll. (2018), Lafont et coll. (2015), Menhour et coll. (2017)]) ${ }^{7}$.

5. Voir, à ce sujet, [Fliess \& Join (2013)] et [Join et coll. (2017)].

6. Acronyme de Multi-Input Multi-Output.

7. Aucun exemple concret n'a été découvert jusqu'à présent où cette approche est prise en défaut. Le «découplage », qui a suscité une recherche aussi abondante que prolixe en automatique théorique, linéaire ou non, est mis en question. Est-il besoin de rappeler une conclusion analogue [Fliess \& Join (2013)] à propos de la « robustesse»? 


\section{Estimation de $F$}

On peut approcher toute fonction $[a, b] \rightarrow \mathbb{R}, a, b \in \mathbb{R}, a<b$, intégrable, c'est-à-dire très générale, par une fonction constante par morceaux (voir, par exemple, [Godement (1998)], [Rudin (1976)]). Par utilisation de fenêtres temporelles glissantes, l'estimation de $F$ se ramène ainsi à celle d'une constante $\Phi$. On présente, pour rester court, les calculs dans le cas $\nu=1$. Les règles du calcul opérationnel (voir, par exemple, [Erdélyi (1962)]) permettent de rééecrire (3) ainsi

$$
s Y=\frac{\Phi}{s}+\alpha U+y(0)
$$

On élimine la condition initiale $y(0)$ en dérivant les deux membres par $\frac{d}{d s}$ :

$$
Y+s \frac{d Y}{d s}=-\frac{\Phi}{s^{2}}+\alpha \frac{d U}{d s}
$$

On atténue le bruit en multipliant les deux membres par $s^{-2}$. La correspondance entre $\frac{d}{d s}$ et le produit par $-t$ permet de revenir au domaine temporel :

$$
F_{\text {est }}(t)=-\frac{6}{\tau^{3}} \int_{t-\tau}^{t}[(\tau-2 \sigma) y(\sigma)+\alpha \sigma(\tau-\sigma) u(\sigma)] d \sigma
$$

où

- $\tau>0$ est « petit» par rapport à la constante de temps la plus rapide ;

- $F_{\text {est }}(t)$ est la valeur numérique constante sur l'intervalle d'intégration attribuée à $F$.

On obtient (9) en temps réel.

On estime $F$, aussi en temps réel, par une formule différente grâce à (8) :

$$
F_{\text {est }}(t)=\frac{1}{\tau}\left[\int_{t-\tau}^{t}\left(\dot{y}^{\star}-\alpha u-K_{P} e\right) d \sigma\right]
$$

Après échantillonnage, (9) et (10) deviennent des filtres linéaires.

Remarque 3. Au début, on estimait F en utilisant un dérivateur [Mboup et coll. (2009)] de y.

Remarque 4. Voir [de Larminat (2009)] et [Carillo \& Rotella (2015)] pour d'autres approches de l'estimation.

\section{2. $A D R C$}

On se conforme à l'usage, contraire à celui du MFC, en présentant l'ADRC par une équation différentielle :

$$
y^{(n)}(t)=f\left(t, y(t), \dot{y}(t), \ldots, y^{(n-1)}(t)\right)+w(t)+a(t, y(t)) u(t)
$$

où

- la fonction $a \neq 0$ est connue ;

- l'ordre de dérivation $n \geqslant 1$ l'est aussi ; 
— la fonction $f$, appelée, parfois, «perturbation interne », ou « endogène », l'est mal ${ }^{8}$;

- $w$ est une «perturbation externe », ou « exogène ».

La some $f+w$ est la «perturbation totale .

En ignorant $w,(11)$ devient :

$$
y^{(n)}(t)=f\left(t, y(t), \dot{y}(t), \ldots, y^{(n-1)}(t)\right)+a(t, y(t)) u(t)
$$

[Sira-Ramírez et coll. (2011)] a observé que (12) vérifie la propriété de platitude et que la sortie y est plate $^{9}$. Si l'on emploie un «correcteur proportionnel intégral généralisé » [Fliess et coll. (2002)], ou «GPI », le « filtrage plat » (flat filtering), [Sira-Ramírez et coll. (2018)], ou les techniques algébriques d'estimation ([Fliess \& Sira-Ramírez (2003), Fliess \& Sira-Ramírez (2008), Fliess et coll. (2008)], et [Sira-Ramírez et coll. (2014)]), l'approche de l'ADRC s'en trouve d'autant plus simplifiée ([Cortés-Romero et coll. (2017), Sira-Ramírez et coll. (2017)]). La distance considérable entre la voie prise par Sira-Ramírez, ses collaborateurs et les autres protagonistes de l'ADRC (voir, en particulier, le livre [Guo \& Zhao (2016)]) rend messéant, on en conviendra, un résumé plus complet.

\section{3. Éléments de comparaison}

\subsection{Prolégomènes}

Posons $\mathfrak{F}=f+w$. Avec cette notation de la perturbation totale, (11) devient

$$
y^{(n)}=\mathfrak{F}+a u(t)
$$

à comparer à (3), si l'on suppose $a$ constant, propriété usuelle, du reste, dans la présentation de l'ADRC :

1. la platitude ne joue aucun rôle en MFC, alors qu'elle est essentielle en ADRC avec, en plus, la nécessité pour $y$ en (12) d'être une sortie plate ${ }^{10}$;

2. en cas de non-platitude, l'ADRC doit, par, exemple se satisfaire du système linéaire variationnel, ou tangent, qui est plat s'il est commandable (voir [Ramírez-Neria et coll. (2014)],

[Sira-Ramírez et coll. (2017)] pour le pendule dit, souvent, de Furuta);

3. $a$ en (11)-(12)-(13) est connu, alors qu' $\alpha$ en (3) est ajusté approximativement par l'opérateur ${ }^{11}$;

4. l'ordre $n$ de dérivation en (11)-(12)-(13) est connu et, donc, imposé, alors que $\nu$ en (3) est choisi par l'opérateur avec, toujours jusqu'à présent, une valeur faible, 1 ou 2.

D'où la sélection d'exemples ci-dessous : ils n'entrent pas dans le cadre a priori de l'ADRC.

\subsection{Un système linéaire}

Pour un système linéaire stationnaire, de dimension finie, commandabilité et platitude sont équivalentes [Fliess et coll. (1995)]. Pour un système SISO, la sortie $y$ est plate si, et seulement si, le numérateur de

8. Cet emploi du mot «perturbation » pour désigner la structure mathématique du système nous semble malheureux.

9. C'est dire, rappelons-le, que $u$ s'exprime comme fonction de $y$ et de ses dérivées jusqu'à un ordre fini. On connaît la richesse de la littérature sur la platitude : voir, par exemple, [Åström \& Murray (2008)], [Lévine (2009)], [Rigatos (2015], [Rotella \& Zambettakis (2007)], [Rudolph (2003)], [Sira-Ramírez \& Agrawal (2004)], et leurs bibliographies.

10. Ce fait est trop souvent ignoré par les tenants de l'ADRC.

11. De façon plus générale, la machinerie pour l'estimation et l'identification semblent moins simple avec l'ARDC. 
la fonction de transfert est constant [Fliess \& Marquez (2000)]. Considérons donc le système défini par la fonction de transfert à numérateur non constant ${ }^{12}$ :

$$
\frac{(s+2)^{2}}{(s-2)(s-1)(s+1)}
$$

Ici, un modèle ultralocal du premier ordre est utilisé avec $\alpha=K_{p}=1$. Un bruit additif, blanc, gaussien, centré, d'écart type 0.01 , affecte la sortie. Les figures 1 et $\mathbf{2}$ présentent les résultats avec une période d'échantillonnage de $T_{e}=10 \mathrm{~ms}$. La poursuite de la trajectoire de référence y est excellente comme le montre le tracé figure 3. Pour cet exemple, contrairement à ce qui suit, on représente la trajectoire par une courbe de Bézier [Bézier (1986), Risler (1991] qui permet aisément des dérivées nulles en début et en fin.

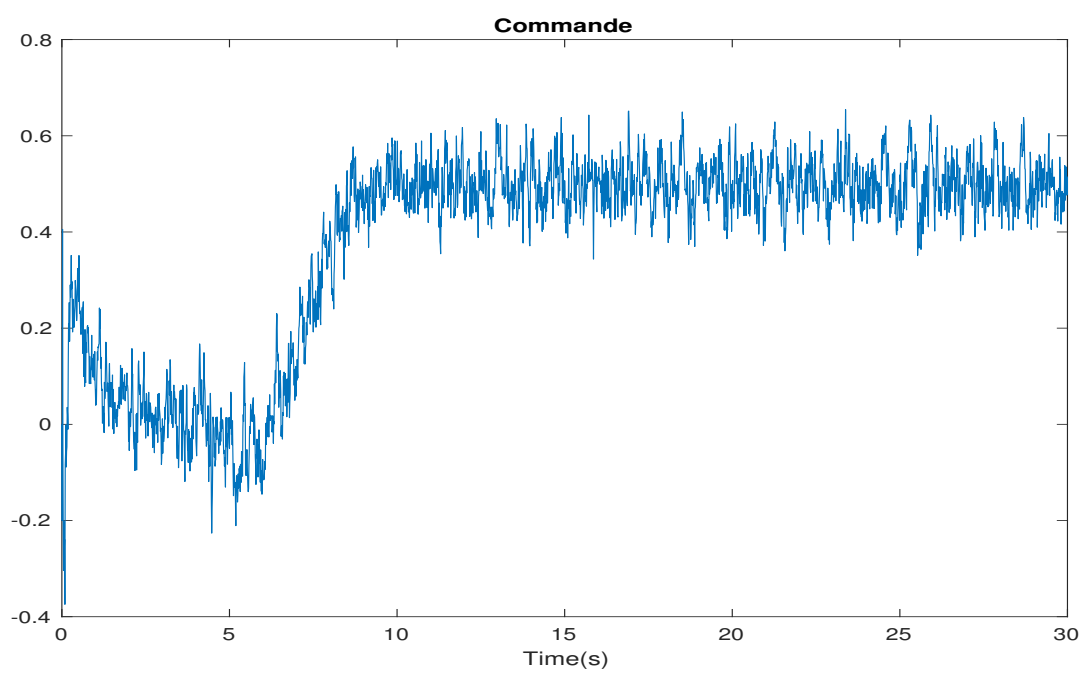

Figure 1.: Commande

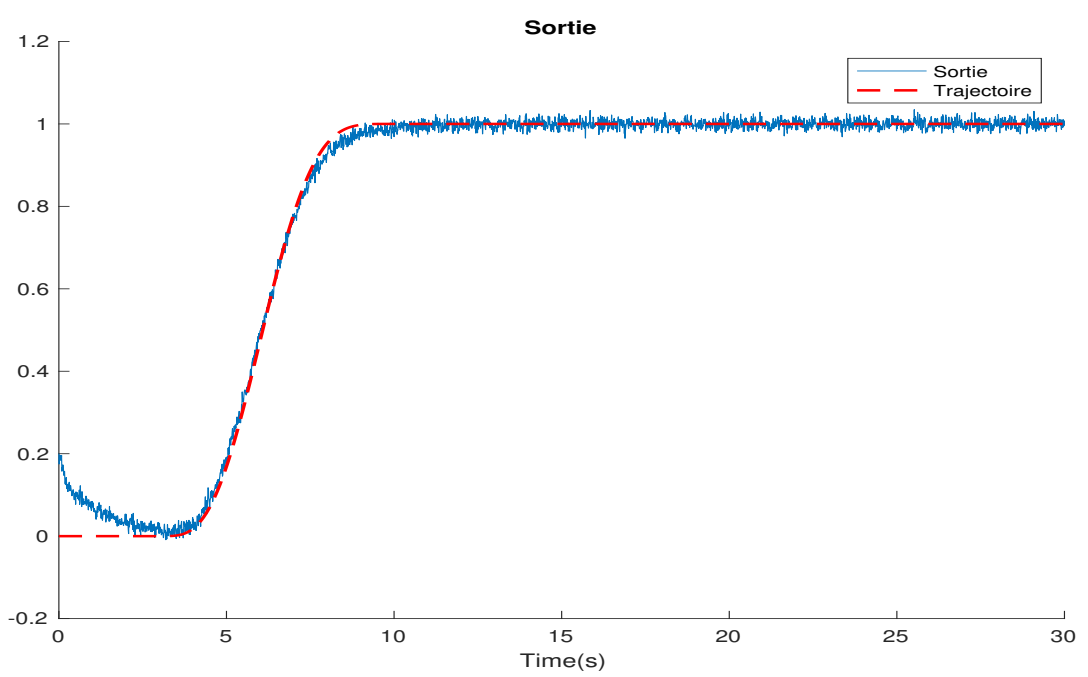

Figure 2.: Sortie

12. Ce cas est d'autant plus éloquent qu'une présentation pour ingénieurs de l'ADRC [Herbst (2013)] ne considère que des fonctions de transfert à numérateurs constants. 


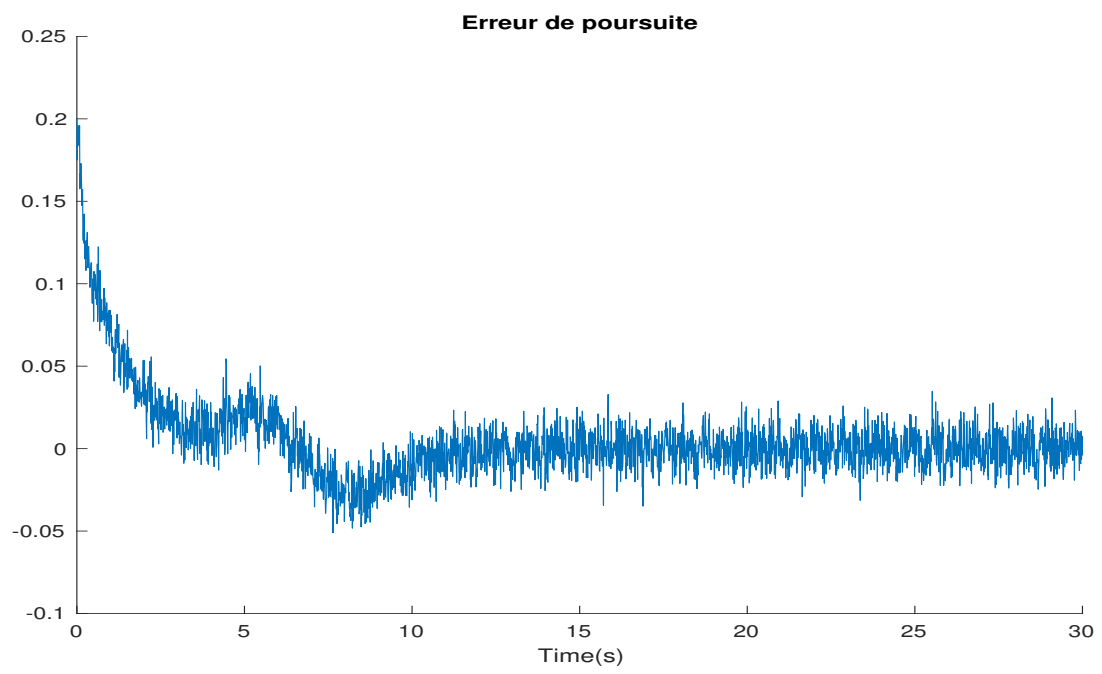

Figure 3.: Erreur de poursuite

\subsection{Cas non linéaires}

Cas 1

Avec

$$
\dot{y}-y=\operatorname{sign}(u) \cdot \sqrt{|u|}
$$

on utilise (3) avec $\nu=1, \alpha=0.1, K_{P}=1$. Un bruit additif, gaussien, centré, d'écart-type 0.05 , affecte la sortie. Avec une période d'échantillonnage $T_{e}=10 \mathrm{~ms}$, les simulations, reportées sur les figures 4, 5 et 6 , attestent d'une poursuite de trajectoire irréprochable.

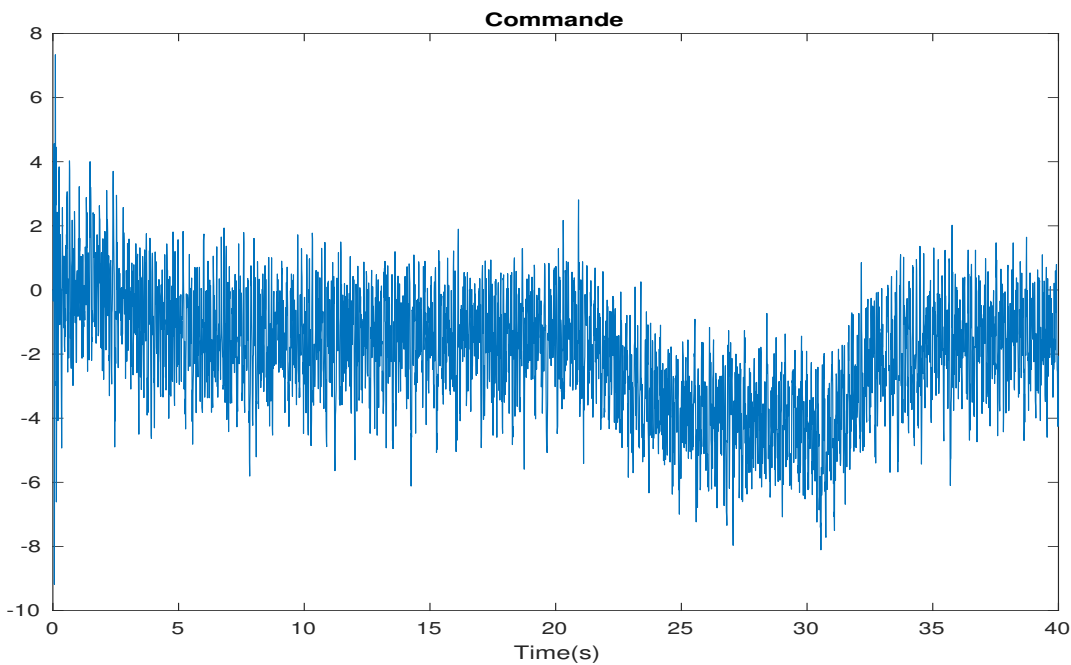

Figure 4.: Commande

Cas 2

Avec

$$
\ddot{y}-1.5 \dot{y}-y=(u+\dot{u})^{3}
$$




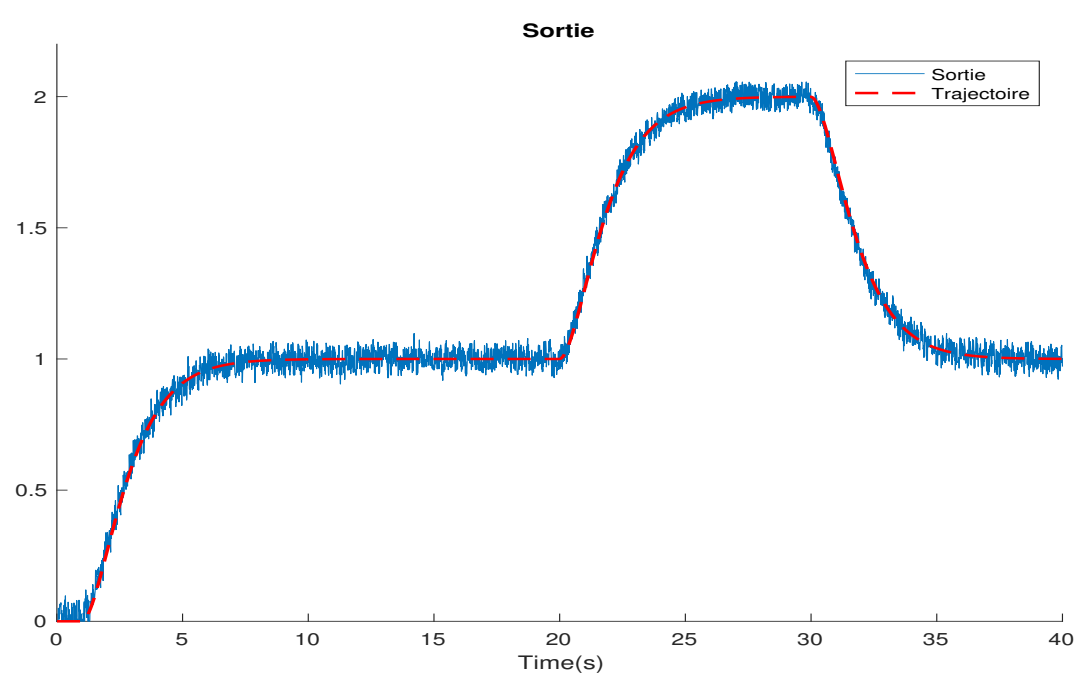

Figure 5.: Sortie

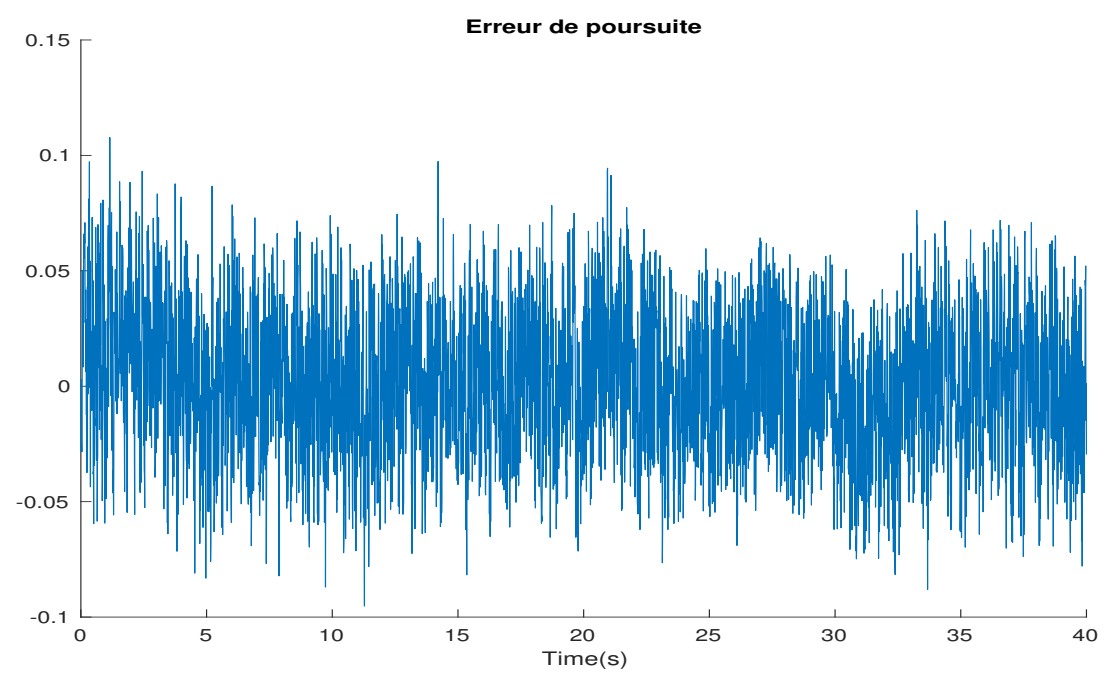

Figure 6.: Erreur de poursuite 
on choisit, pour (3), $\nu=1, \alpha=K_{P}=10$. Avec un bruit sur $y$, additif, blanc, gaussien, centré, d'écarttype 0.01 , et un échantillonnage de période $T_{e}=10 \mathrm{~ms}$, la poursuite de trajectoire, exhibée par les figures 7,8 et 9 est bonne.

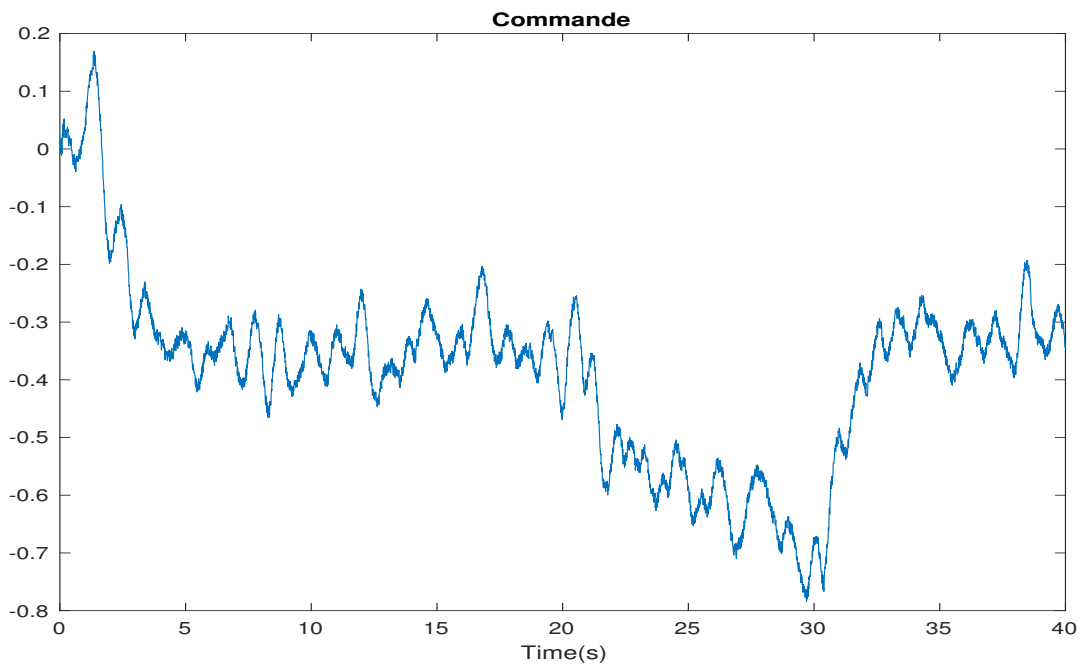

Figure 7.: Commande

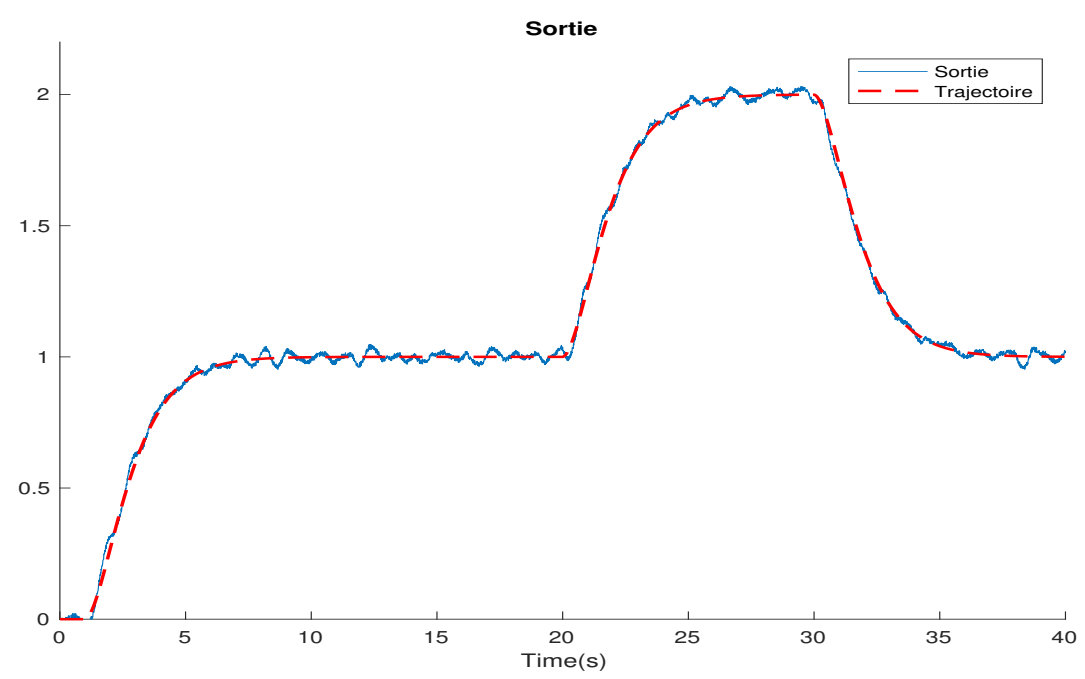

Figure 8.: Sortie

Cas 3

Avec

$$
\ddot{y}+3 \dot{y}+2 y=\operatorname{sign}(u) \cdot 10^{|u|}
$$

$\nu=1, \alpha=10, K_{P}=1$ en (3). Les résultats des figures 10,11 et 12 sont excellents. 


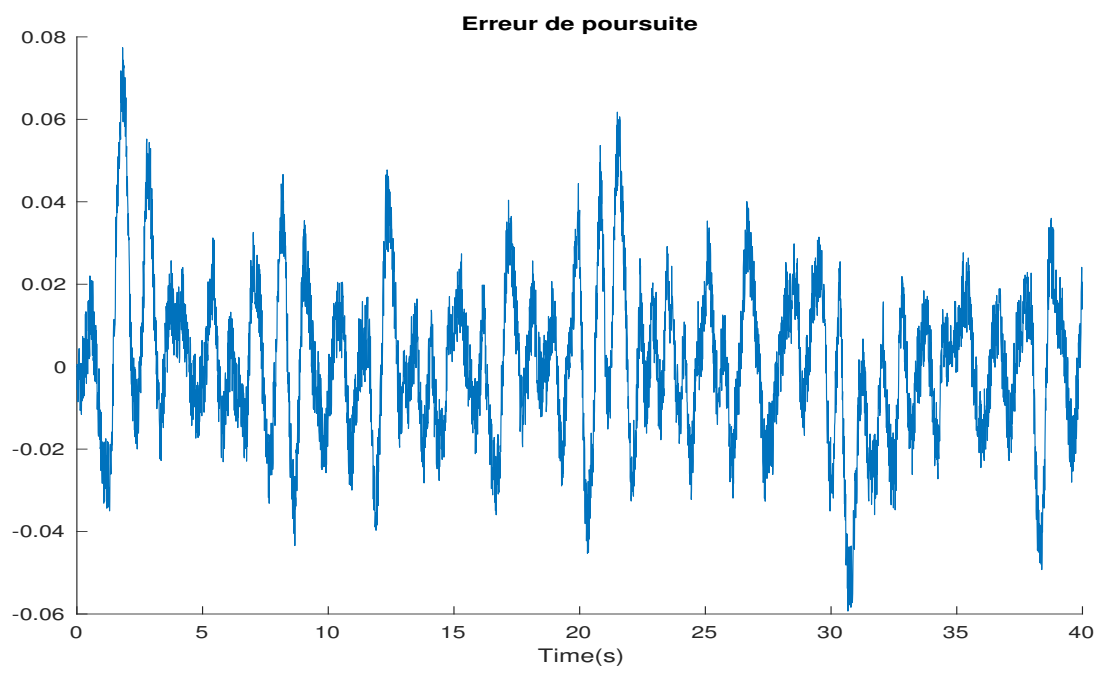

Figure 9.: Erreur de poursuite

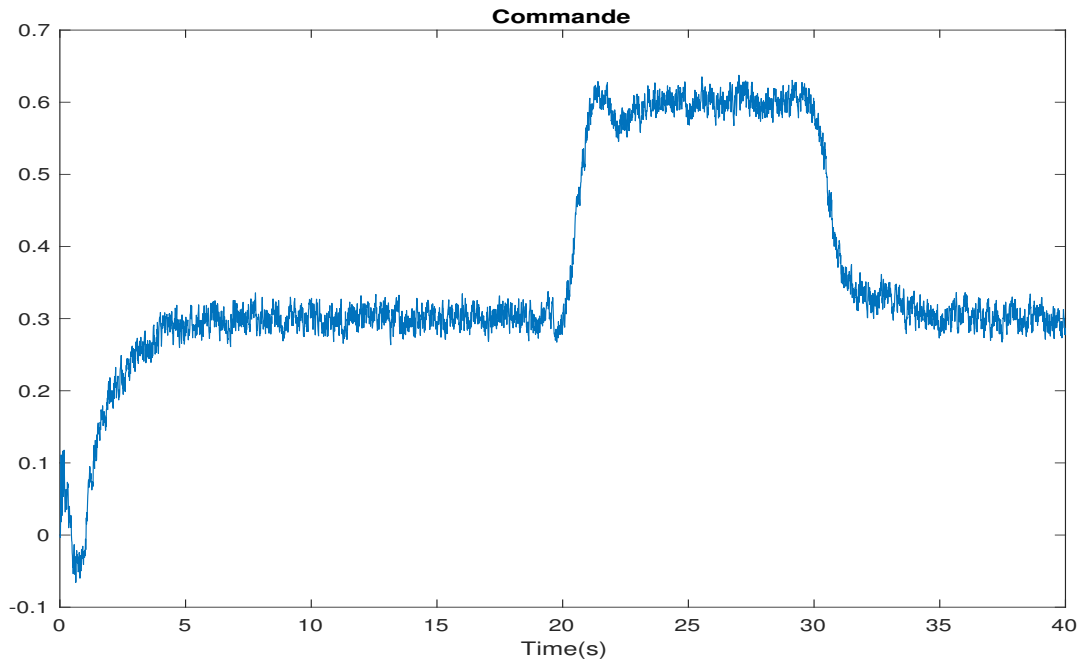

Figure 10.: Commande

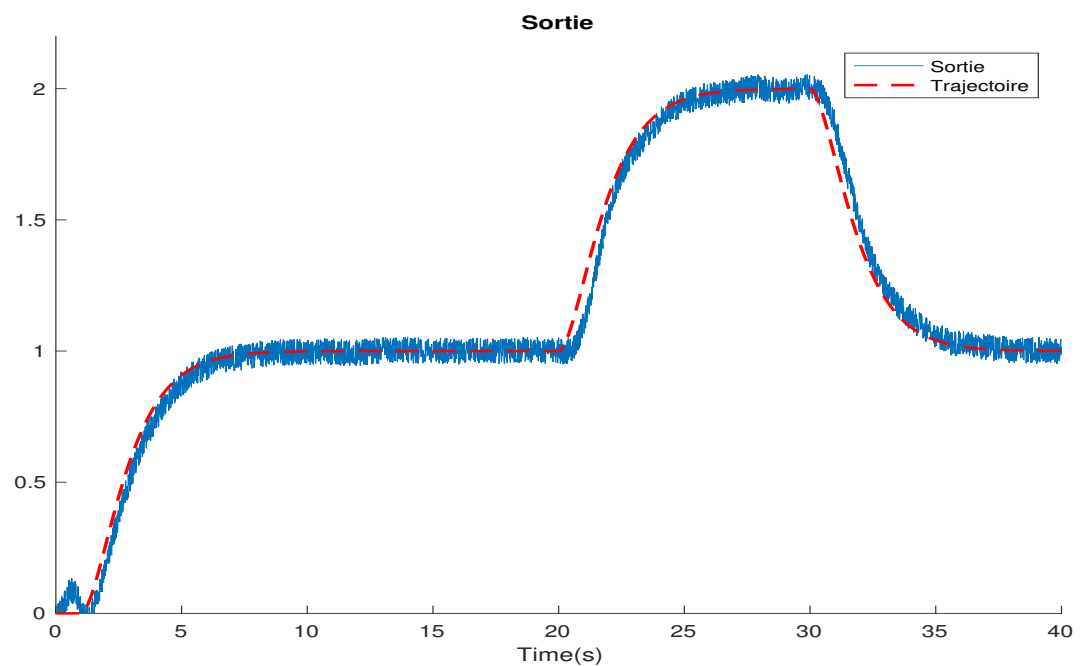

Figure 11.: Sortie 


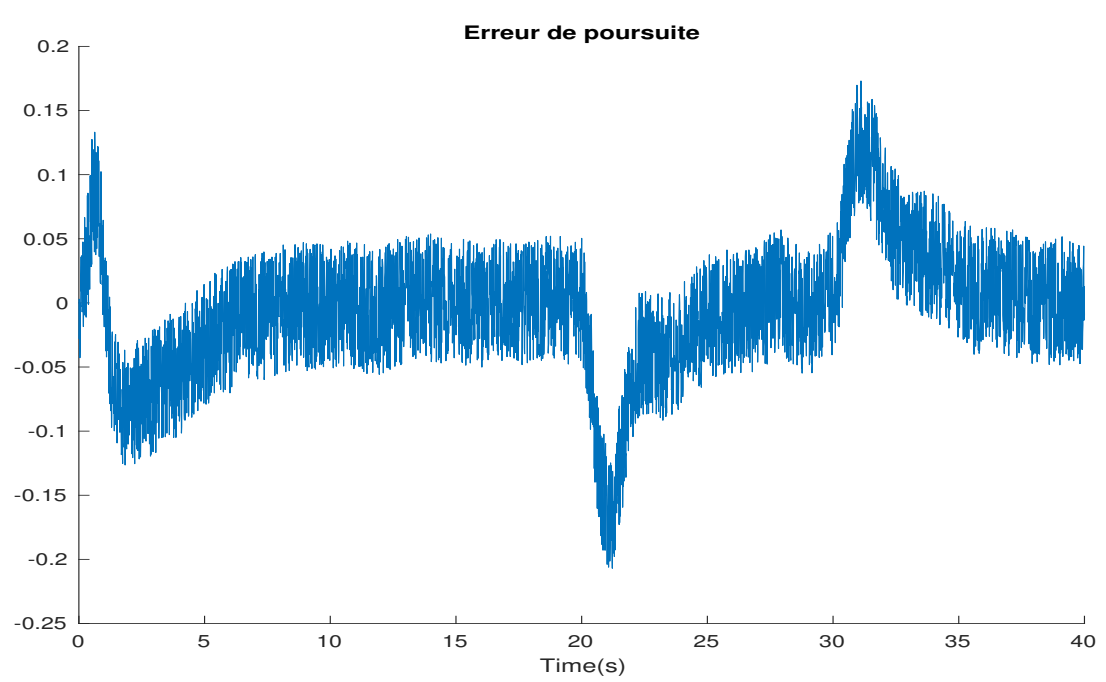

Figure 12.: Erreur de poursuite

Cas 4

Quoique le système

$$
\dot{y}-y=(0.5 \dot{y}+y) u
$$

soit plat, de sortie plate $y$, il est, en raison du produit $\dot{y} u$ et comme on le voit en (12), plus général que ceux considérés en ADRC. De plus, son instabilité le rend délicat à commander. En (3), on choisit $\nu=1$, $\alpha=5, K_{P}=3$. On ajoute un bruit additif sur la sortie, blanc, gaussien, centré, d'écart-type 0.05. La condition initiale est $y(0)=0.2$. La période d'échantillonnage est $T_{e}=10 \mathrm{~ms}$. Les résultats de poursuite de trajectoire reportés sur les figures 13,14 et 15 sont tout à fait corrects.

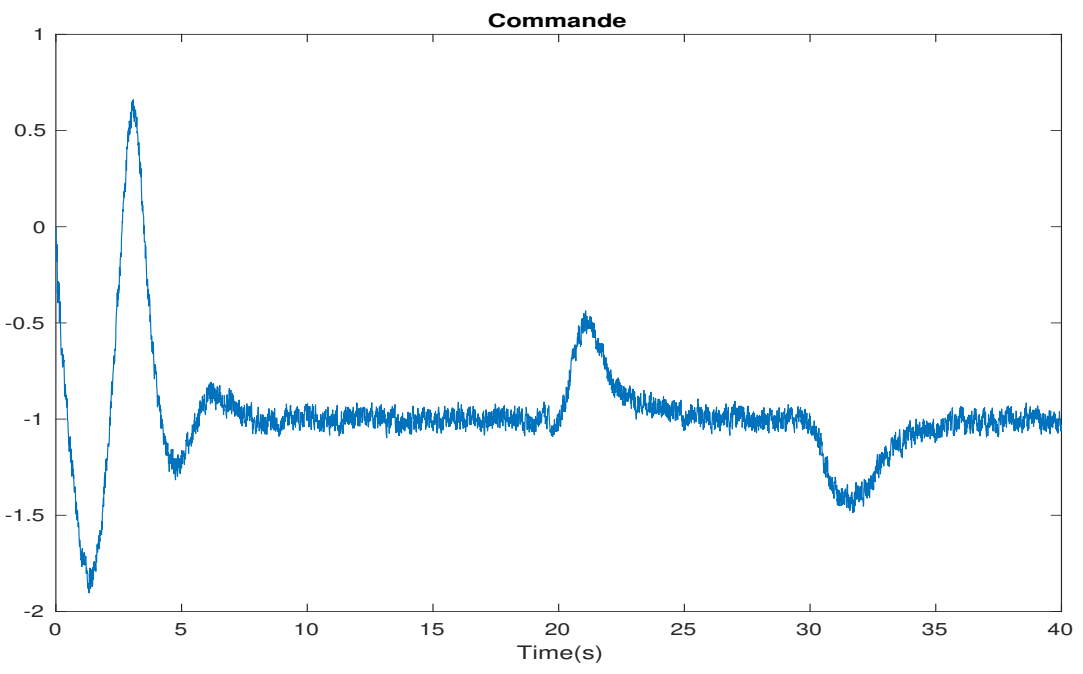

Figure 13.: Commande

\section{Cas 5}

Une littérature considérable a été consacrée au système, plutôt académique, des trois cuves, notamment en diagnostic, représenté par la figure 16 (voir, par exemple, [Noura et coll. (2009)] et ses ré- 


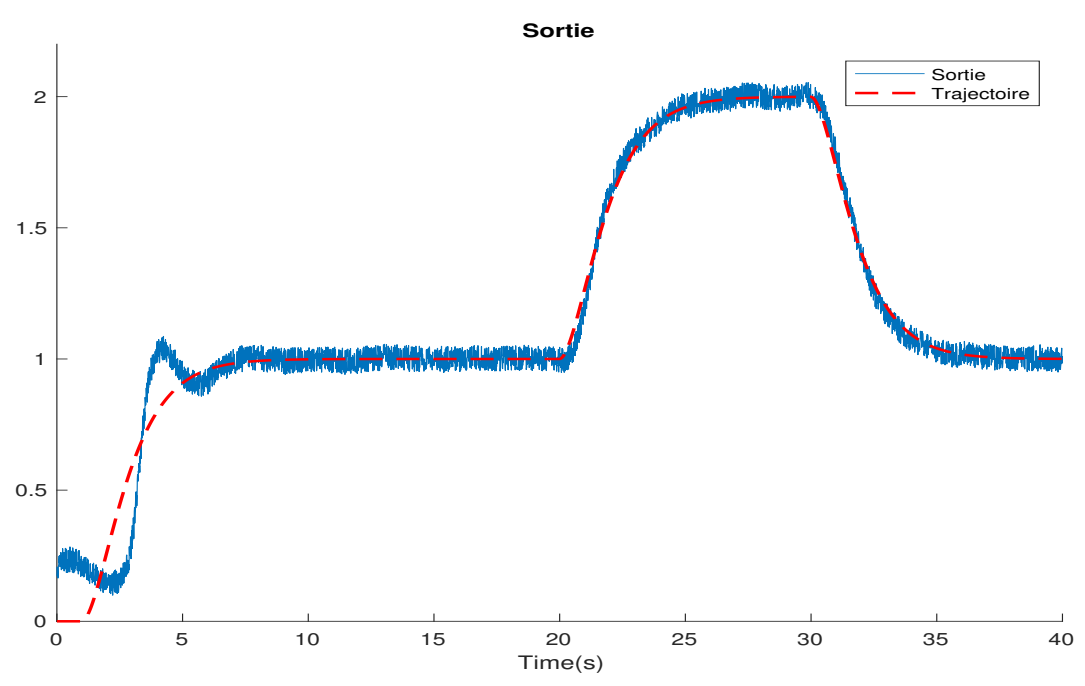

Figure 14.: Sortie

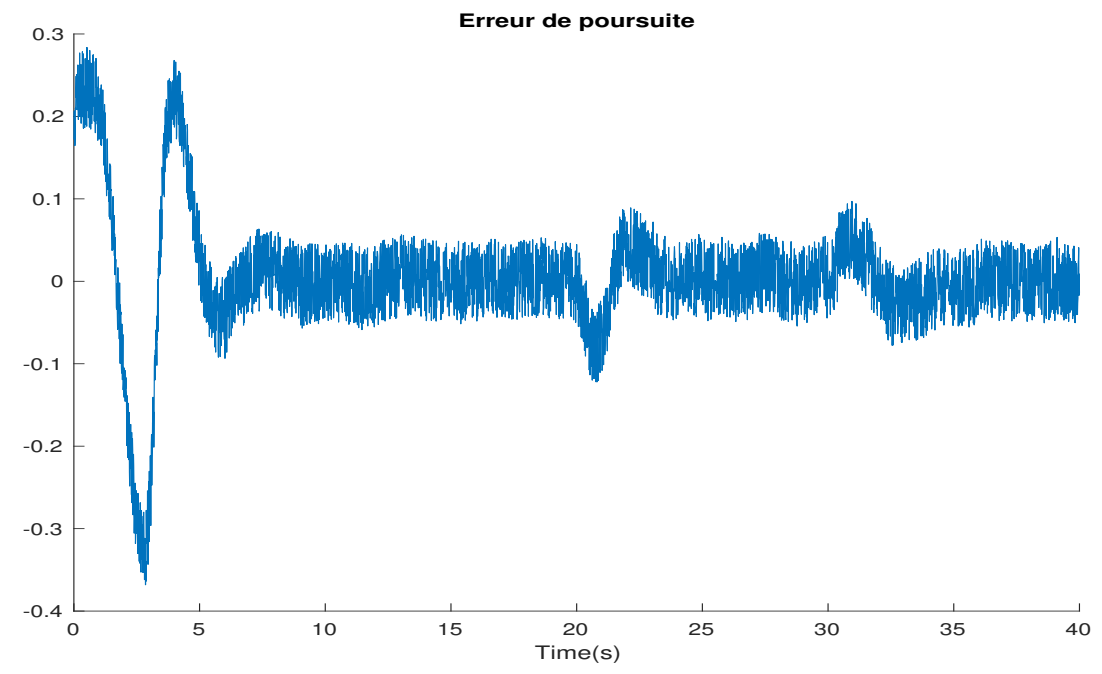

Figure 15.: Erreur de poursuite 
férences). Confirmant la remarque 2, il témoigne de la simplicité du traitement des systèmes mul-

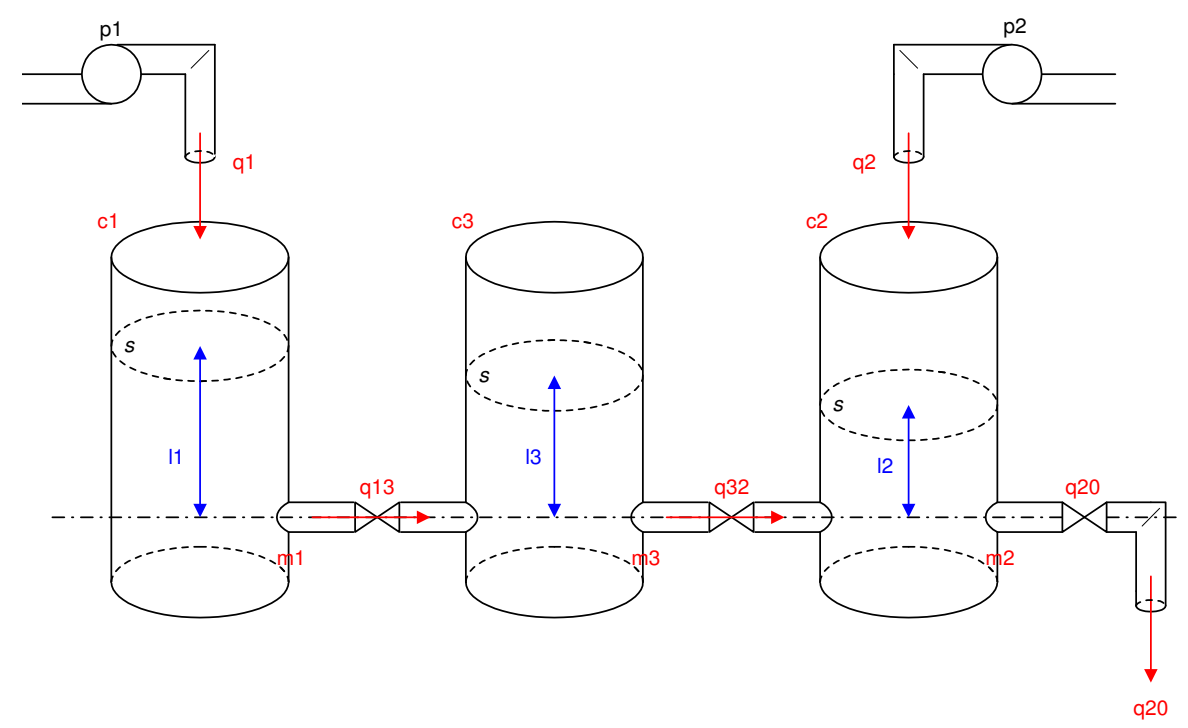

Figure 16.: Système des trois cuves

tivariables par rapport à l'ADRC (voir, par exemple, [Guo \& Zhao (2016)], [Inoue \& Ishida (2016)], [Sira-Ramírez et coll. (2017)], et [Sun et coll. (2016)]). En voici la description par équations différentielles

$$
\left\{\begin{array}{l}
\dot{x}_{1}=-D \mu_{1} \operatorname{sign}\left(x_{1}-x_{3}\right) \sqrt{\left|x_{1}-x_{3}\right|}+u_{1} / S \\
\dot{x}_{2}=D \mu_{3} \operatorname{sign}\left(x_{3}-x_{2}\right) \sqrt{\left|x_{3}-x_{2}\right|}-D \mu_{2} \operatorname{sign}\left(x_{2}\right) \sqrt{\left|x_{2}\right|}+u_{2}(t) / S \\
\dot{x}_{3}=-D \mu_{1} \operatorname{sign}\left(x_{1}-x_{3}\right) \sqrt{\left|x_{1}-x_{3}\right|} D \mu_{3} \operatorname{sign}\left(x_{3}-x_{2}\right) \sqrt{\left|x_{3}-x_{2}\right|} \\
y_{1}=x_{1} \\
y_{2}=x_{2} \\
y_{3}=x_{3}
\end{array}\right.
$$

où

- $x_{i}, i=1,2,3$, est le niveau de liquide dans la cuve $i$,

- les commandes $u_{1}, u_{2}$ sont les arrivées de liquide,

$-D=\left(S_{p} \sqrt{2 g}\right) / S$,

- $g=9.81 \mathrm{~m} \cdot \mathrm{s}^{-2}$ est la pesanteur,

- Les valeurs numériques suivantes sont empruntées à une maquette de laboratoire :

$-S=0.0154 \mathrm{~m}$ est la section des cuves,

$-S_{p}=5.10^{-5} \mathrm{~m}$ est la section des tuyaux entre cuves,

$-\mu_{1}=\mu_{3}=0.5, \mu_{2}=0.675$ sont les coefficients de viscosité.

On régule, selon le paragraphe 2.1., grâce à deux systèmes ultralocaux (3) en parallèle, d'ordre 1 et monovariables, de commandes et de sorties respectives $u_{1}, u_{2}$ et $y_{1}, y_{2}$. Pour les deux, $\alpha=100, K_{P}=0.5$. Un bruit additif, blanc, gaussien, centré, d'écart-type $0,5.10^{-3}$, parasite les sorties. Les figures 17 à 21 rapportent les simulations obtenues avec un échantillonnage d'1s. Les poursuites de trajectoires sont excellentes (se rapporter à 19 et 22). En dépit du couplage physique évident, une variation de consigne dans la cuve 1 n'influence guère la poursuite dans la cuve 2 . 


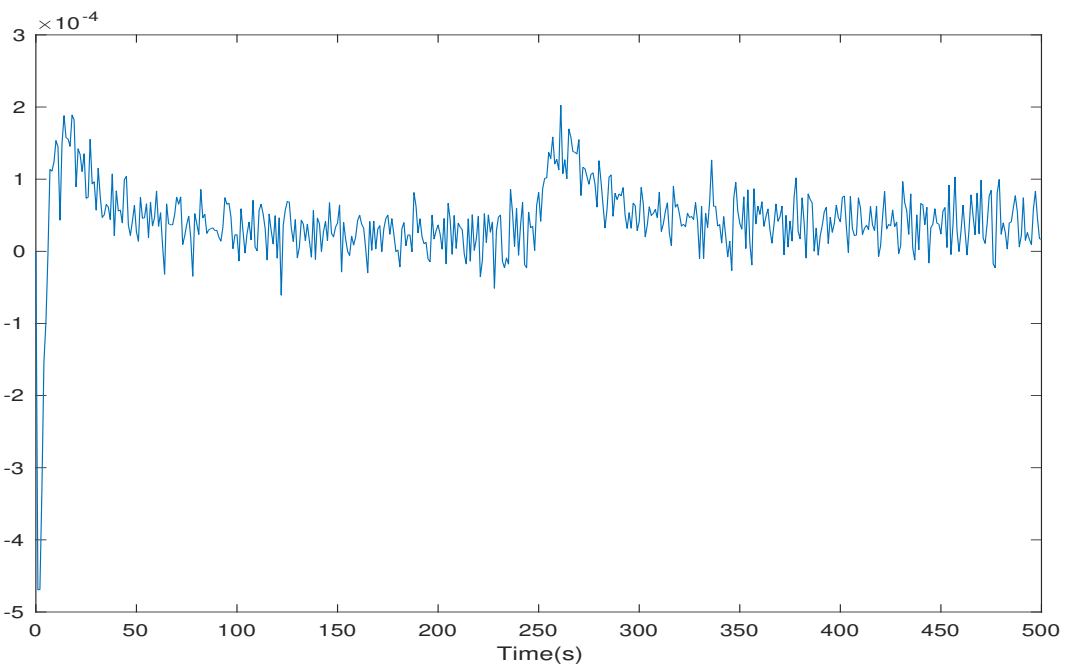

Figure 17.: Commande de la pompe 1

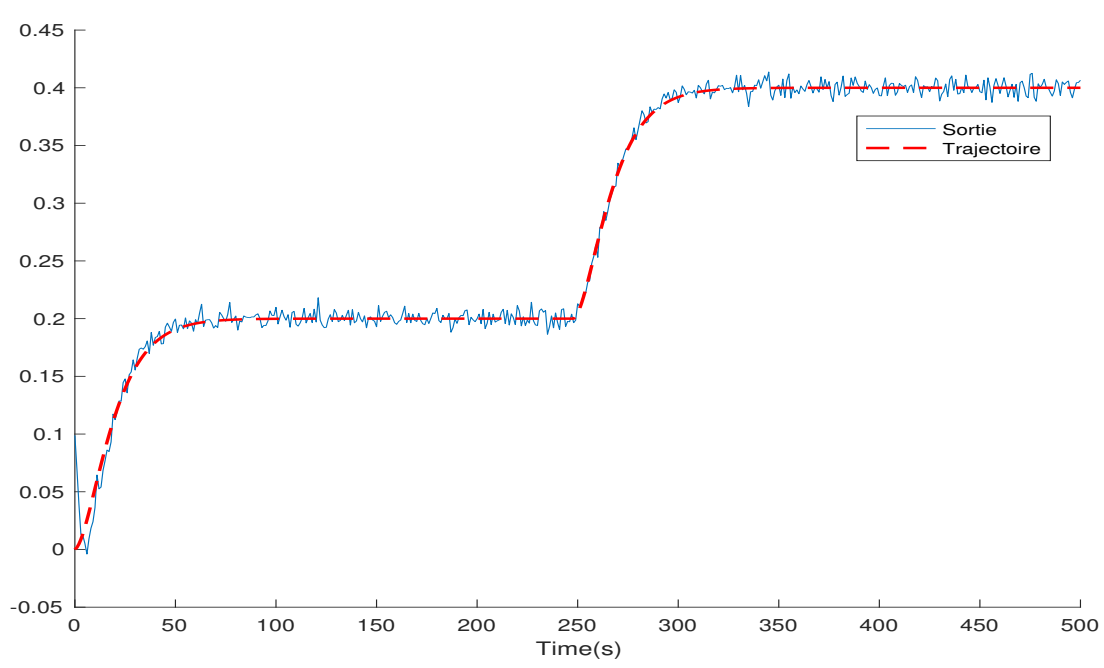

Figure 18.: Evolution du niveau dans la cuve 1

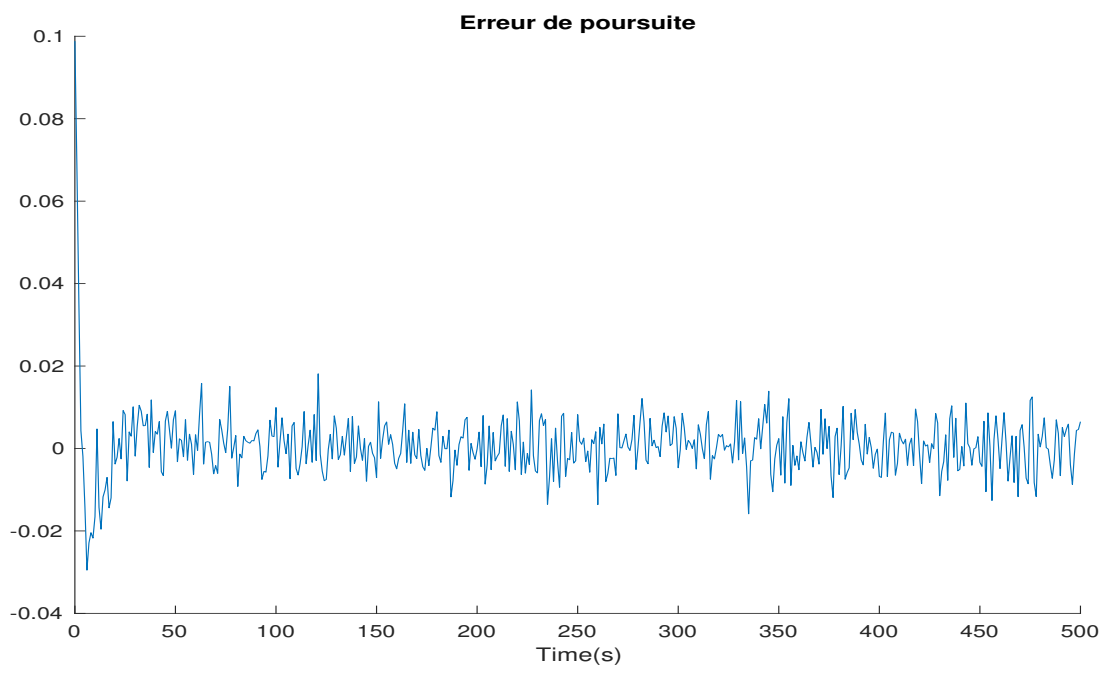

Figure 19.: Erreur de poursuite pour la cuve 1 


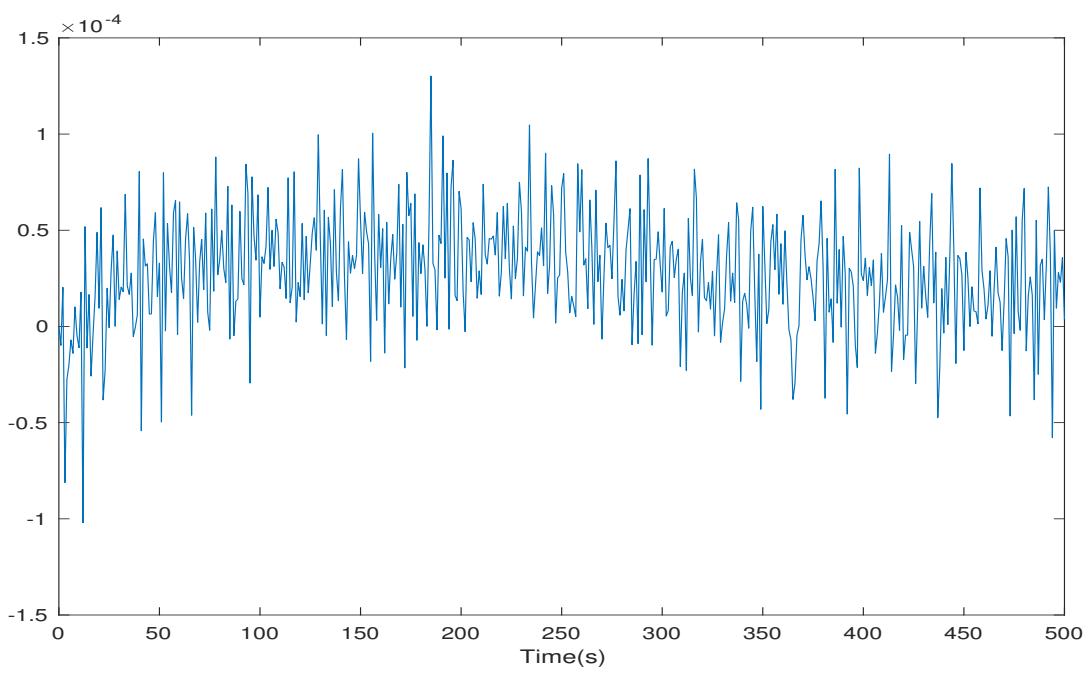

Figure 20.: Commande de la pompe 2

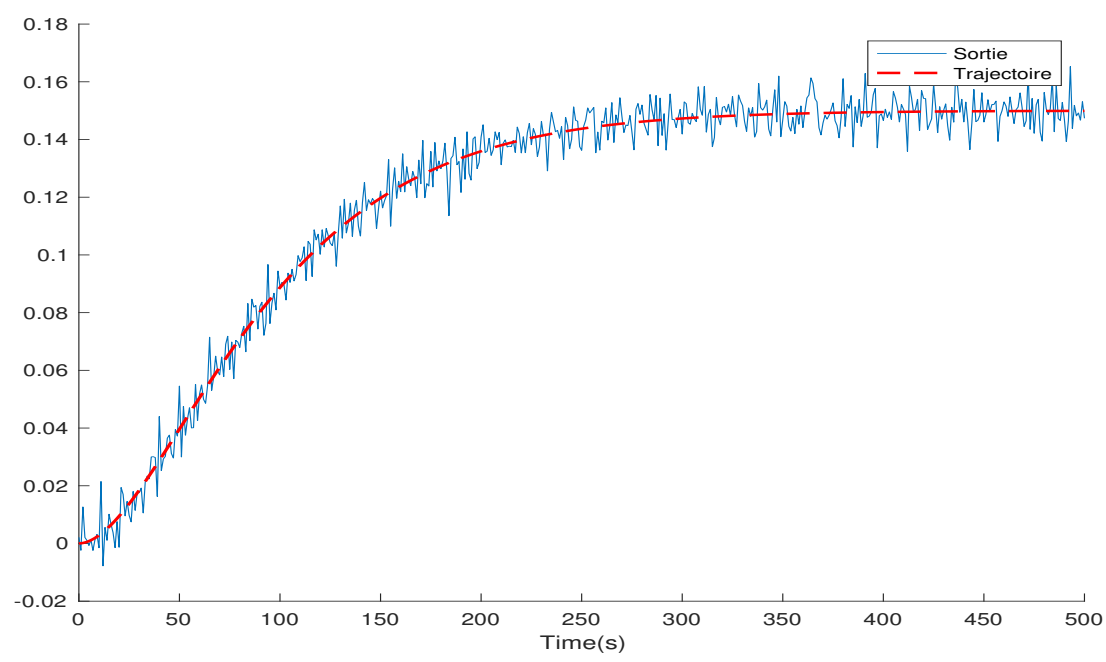

Figure 21.: Evolution du niveau dans la cuve 2

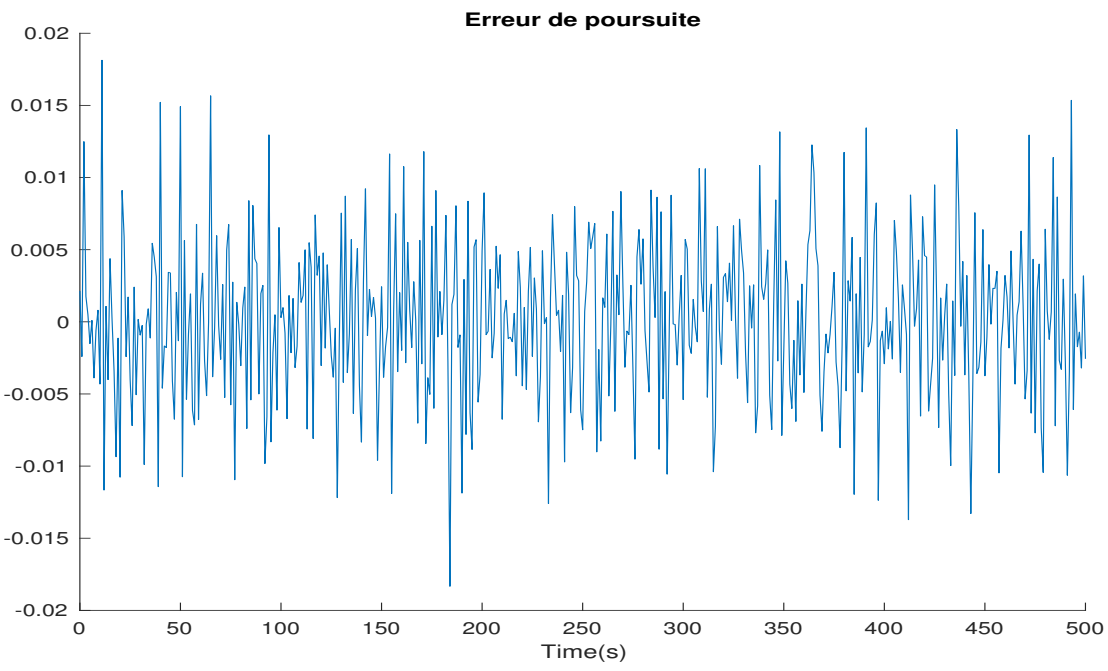

Figure 22.: Erreur de poursuite pour la cuve 2 
3.4. Paramètres répartis : une équation aux dérivées partielles

Soit l'équation de la chaleur en une seule dimension d'espace

$$
\frac{\partial w}{\partial t}=\frac{\partial^{2} w}{\partial x^{2}}
$$

où

$-0 \leqslant x \leqslant 1$;

- $w(t, 1)=u(t)$ est la commande;

- $w(t, 0)=0.5$ est la condition à l'autre bord ;

$-w(0, x)=0.5+(u(0)-0.5) x$ est la condition initiale.

On désire maîtriser l'évolution de $w(t, x)$ en $x=1 / 3$ (voir figure 24). Ici, $\nu=1, \alpha=K_{P}=10$. Réalisées avec un bruit additif, blanc, gaussien, centré, d'écart type 0.01, et un échantillonnage de taille $T_{e}=10 \mathrm{~ms}$, les simulations des figures 23, 24, 25 et 26 présentent d'excellents résultats.

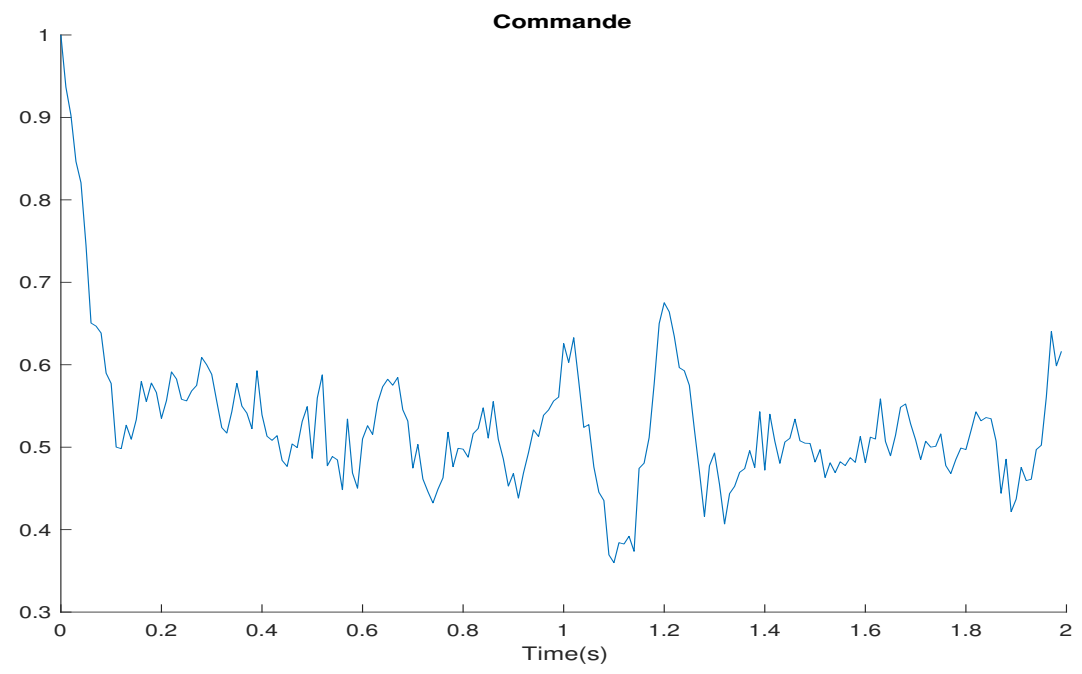

Figure 23.: Commande

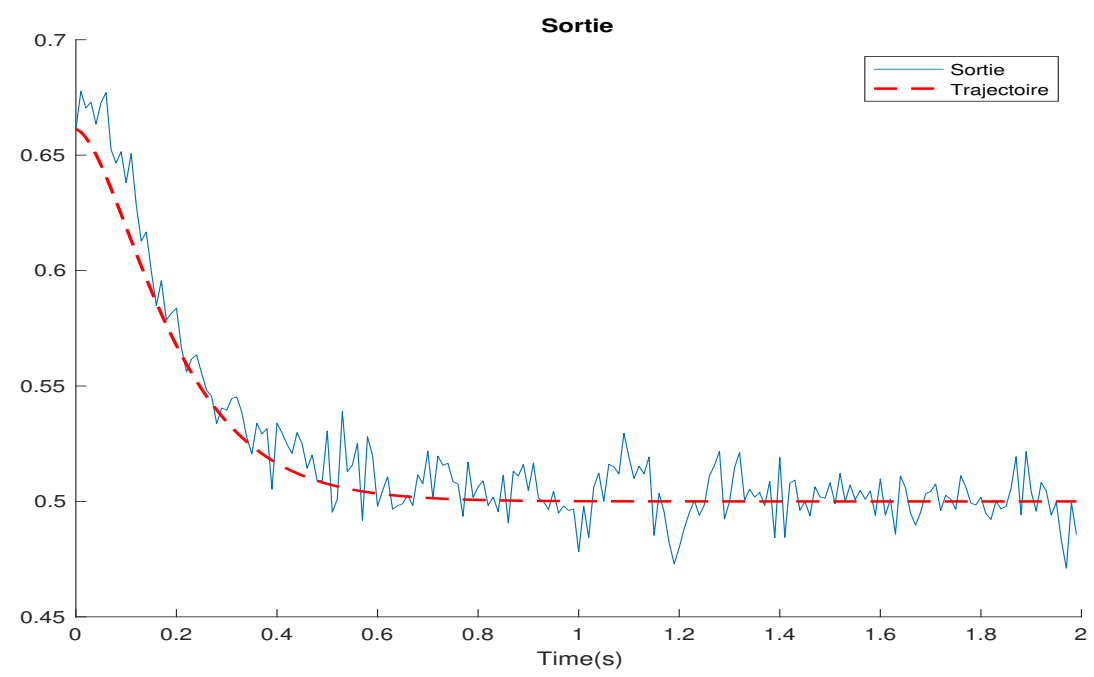

Figure 24.: Sortie 


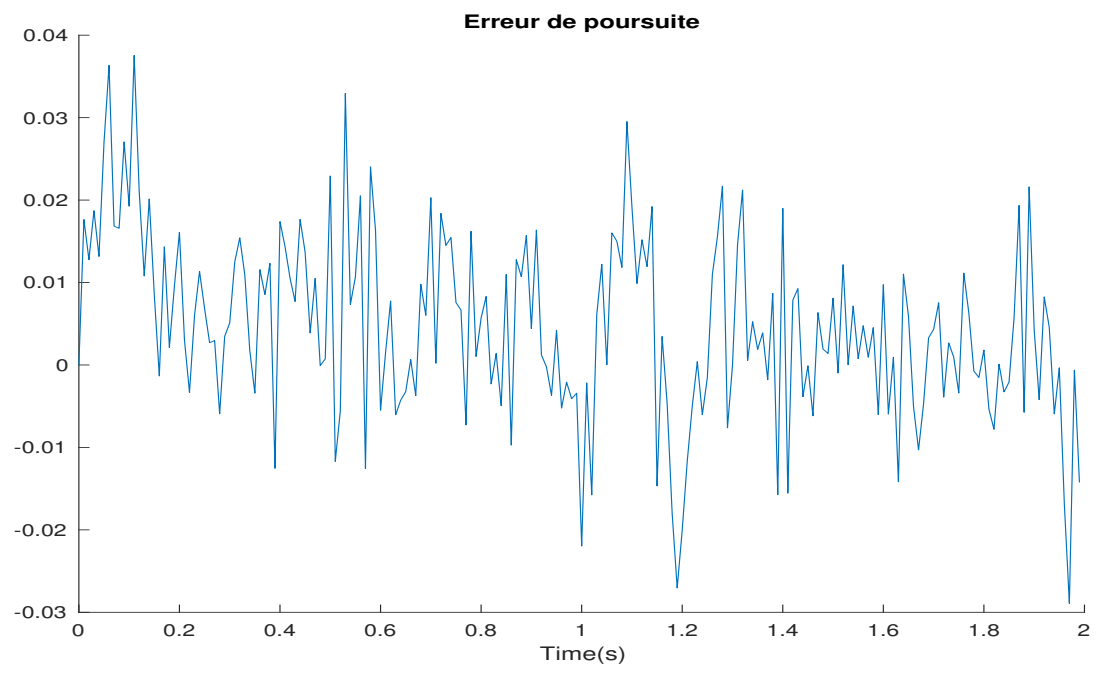

Figure 25.: Erreur de poursuite

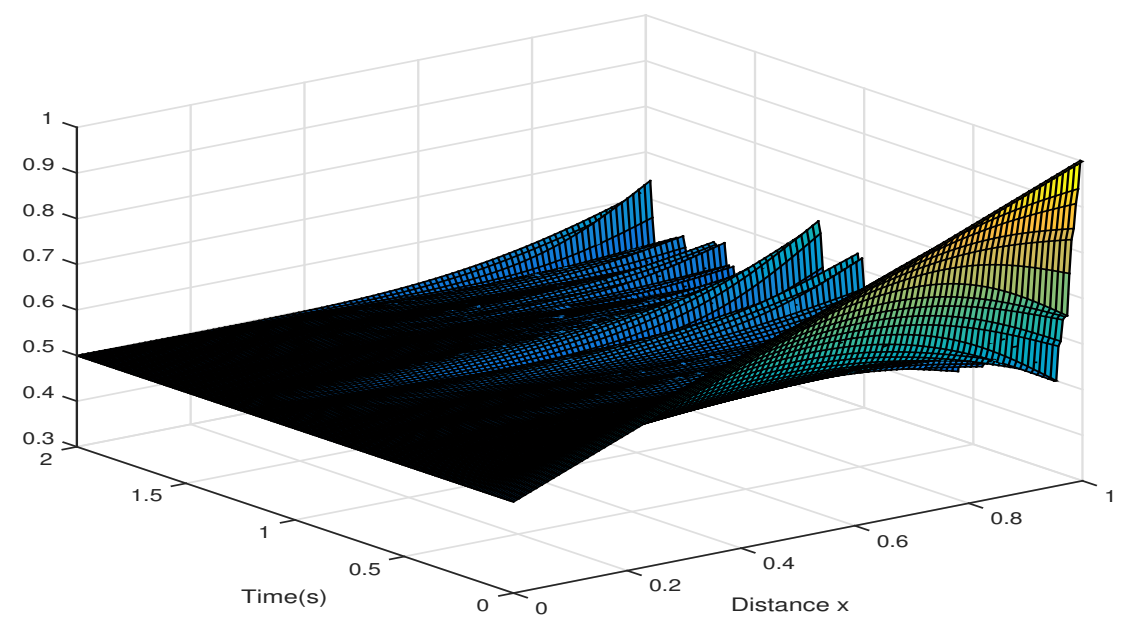

Figure 26.: Evolution de la température de poutre 
Remarque 5. En calcul opérationnel (voir, par exemple, [Erdélyi (1962)]), (15) conduit à considérer des exponentielles de la forme $\exp ( \pm x \sqrt{s})$. Nul besoin avec MFC de développements originaux pour la dérivation fractionnaire $s^{1 / 2}$ et, on en fait la conjecture, pour toute dérivation non entière rencontrée en pratique. Ce n'est pas le cas avec l'ADRC (voir, par exemple, [Li et coll. (2016)]).

\section{Conclusion}

Le MFC a déjà permis des avancées conceptuelles d'une importance certaine :

1. On fournit une explication, en [d'Andréa-Novel et coll. (2010)], [Fliess \& Join (2013)], à l'universalité des PID et à leurs faiblesses, et ce pour la première fois.

2. En dépit de performances parfois insatisfaisantes, ALINEA ${ }^{13}$ est l'algorithme le plus usité de régulation des accès d'autoroutes. Aucune justification n'en avait été publiée jusqu'à [Abouaïssa et coll. (2017a)].

3. Réponse [Fliess \& Join (2018)] à une question [Karin et coll. (2016)] sur la « compensation dynamique ", en «biologie systémique », ou systems biology.

Aucun succès de cet ordre ne peut, à notre connaissance, être porté au crédit de l'ADRC.

Depuis [Ziegler \& Nichols (1942), Ziegler \& Nichols (1943)], des retards sont introduits pour faciliter le réglage des gains des PID. Ils perdent tout sens [Fliess \& Join (2013)] avec les correcteurs intelligents associés au MFC. Avec, par contre, des retards physiques « assez importants ", aucune solution définitive ne semble s'imposer à l'heure où ces lignes sont écrites, en dépit d'avancées prometteuses, tant en MFC (voir [Doublet et coll. (2016)],

[Doublet et coll. (2017)], où $\alpha$ en (3) devient variable, et [Thabet et coll. (2017)],

[Zhang et coll. (2018)]) qu'en ADRC (voir, par exemple, [Guo \& Zhao (2016)],

[Sira-Ramírez et coll. (2017)], [Xia \& coll. (2009)], [Zhao \& Gao (2014)]). Dominer cette question est un point clé.

Quant aux systèmes a priori modélisés par des équations aux dérivées partielles, la situation reste assez floue, même si MFC compte déjà quelques réussites concrètes incontestables (voir, par exemple, [Join et coll. (2010)] ${ }^{14}$ ).

\section{Bibliographie}

AbOuAïSSA H., FliESS M., JoIN C., «On ramp metering : Towards a better understanding of ALINEA via model-free control ». International Journal of Control, 90 (2017a) : 1018-1026.

Abouaïssa H., Alhaj Hasan O., Join C., Fliess M., Defer D., Energy saving for building heating via a simple and efficient model-free control design : First steps with computer simulations. 21st International Conference on System Theory, Control and Computing, Sinaia, 2017b.

https://hal.archives-ouvertes.fr/hal-01568899/en/

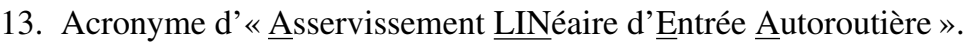

14. Il s'agit d'une installation hydroélectrique, souvent modélisée, comme les canaux, par une équation de Saint-Venant. La solution proposée, brevetée par EDF (Électricité de France) et l'École polytechnique, a reçu le prix de l'innovation 2010, catégorie «brevet », décerné par l'École polytechnique. 
Aguilar-Ibañez C., SiRA-RamíRez H., Acosta J. Á, « Stability of active disturbance rejection control for uncertain systems: A Lyapunov perspective ». International Journal of Robust and Nonlinear Control, (2017) : DOI : $10.1002 / \mathrm{rnc} .3812$

D'AndréA-Novel B., Fliess M., Join C., Mounier H., Steux B., «A mathematical explanation via "intelligent" PID controllers of the strange ubiquity of PIDs ". 18th Mediterranean Conference on Control \& Automation, Marrakech, 2010. https://hal .archives-ouvertes.fr/inria-00480293/en/

Åström K.J., HäGGLUnd T., Advanced PID Control. Research Triangle Park, NJ : Instrument Society of America, 2006.

Åström K.J. , Kumar P.R., « Control: A perspective ». Automatica, 50 (2014) : 3-43.

Åström K.J., Murray R.M., Feedback Systems : An Introduction for Scientists and Engineers. Prinveton, NJ : Princeton University Press, 2008.

BEKCHEVA M., JOIN C., MOUNIER H., «Quasi-model-free tracking of quadrotors », soumis (2018).

BÉZIER P., Courbes et surfaces. Paris : Hermès, 1986.

CARILLO F., ROTELLA F., « Some contributions to estimation for model-free control ». IFAC-PapersOnLine 48-28 (2015) : 150-155.

Choquet G, (2000), Cours de topologie. Paris : Dunod, 2000.

Cortés-Romero J., Jimenez-Triana A., Coral-Enriquez H., Sira- Ramírez H., « Algebraic estimation and active disturbance rejection in the control of flat systems ». Control Engineering Practice, 61 (2017) : 173182.

Doublet M., Join C., Hamelin F., « Model-free control for unknown delayed systems ». 3rd International Conference on Control and Fault-Tolerant Systems, Barcelone, 2016.

https://hal.archives-ouvertes.fr/hal-01369776/en/

DOUBLET M., JoIN C., HAMELIN F., « Stability analysis for unknown delayed systems controlled by model-free control ». 21st International Conference on System Theory, Control and Computing, Sinaia, 2017.

https://hal.archives-ouvertes.fr/hal-01578561/en/

ERdÉLYI A., Operational Calculus and Generalized Functions. New York : Holt Rinehart Winston, 1962.

FENG H., GuO B.-Z., « Active disturbance rejection control: Old and new results ». Annual Reviews in Control, (2017) : http://dx.doi.org/10.1016/j.arcontrol.2017.05.003

FLIESS M., JOIN C., « Model-free control ». International Journal of Control, 86 (2013) : 2228-2252.

FLIESS M., JOIN C., «Dynamic compensation and homeostasis: a feedback control perspective 》 (2018). https://hal .archives-ouvertes.fr

Fliess M., JoIN C., SiRA-RamíREZ H., « Non-linear estimation is easy ». International Journal of Modelling Identification and Control, 4 (2008) : 12-27.

FLIESS M., LAMNABHI M., LAMNABHI-LAGARRIGUE F., «An algebraic approach to nonlinear functional expansions ». IEEE Transactions on Circuits and Systems, 30 (1983) : 554-570.

Fliess M., LÉVINE J., MARTIN Ph., ROUCHON P., «Flatness and defect of non-linear systems: introductory theory and examples».

International Journal of Control, 61 (1995) : 1327-1361.

Fliess M., LÉVINE J., MARTIN Ph., ROUCHON P., «A Lie-Bäcklund approach to equivalence and flatness of nonlinear systems ». IEEE Transactions on Automatic Control, 44 (1999) : 922-937.

Fliess M., MARQUez R., "Continuous-time linear predictive control and flatness: a module-theoretic setting with examples ». International Journal of Control, 73 (2000) : 606-623.

Fliess M., Marquez R., Delaleau E., Sira-Ramírez H., "Correcteurs proportionnels intégraux généralisés ». ESAIM: Control Optimisation and Calculus of Variations, 7 (2002) : 23-41.

Fliess M., SiRA-RAmíREZ H., «An algebraic framework for linear identification ». ESAIM: Control Optimisation and Calculus of Variations, 9 (2003) : 151-168.

Fliess M., SiRA-RAmíREZ H., "Closed-loop parametric identification for continuous-time linear systems via new algebraic techniques ». H. Garnier, L. Wang (Eds): Identification of Continuous-time Models from Sampled Data, pp. 362-391, Londres : Springer, 2008. 
GAO Z., «On the centrality of disturbance rejection in automatic control ». ISA Transactions, 53 (2014) : 850857.

Godement R., Analyse mathématique II. Berlin : Springer, 1998.

Guo B.-Z., ZHAO Z.-L., Active Disturbance Rejection Control for Nonlinear Systems: An Introduction. Singapore : Wiley, 2016.

GuO B.-Z., ZHOU H.-C., «The active disturbance rejection control to stabilization for multi-dimensional wave equation with boundary control matched disturbance ». IEEE Transactions on Automatic Control, 60 (2015) : 143-157.

HAN J., « From PID to active disturbance rejection control ». IEEE Transactions on Industrial Electronics, 56 (2009) : 900-906.

HERBST G., «A simulative study on active disturbance rejection control (ADRC) as a control tool for practitioners ». Electronics, 2 (2013) : 246-279.

HUANG Y., XUE W., «Active disturbance rejection control: Methodology and theoretical analysis ». ISA Transactions, 53 (2014) : 963-976.

INOUE S., ISHIDA Y., «Design of a model-following controller using a decoupling active disturbance rejection control method ». Journal of Electrical \& Electronic Systems, 5 (2016) : doi:10.4172/2332-0796.1000174

Join C., Delaleau E., Fliess M., Moog C.H., «Un résultat intrigant en commande sans modèle ». ISTE OpenScience Automatique, 1 (2017) : 9 p. https: / / hal .archives-ouvertes.fr/hal-01628322/en/

JoIN C., Robert G., Fliess M., « Vers une commande sans modèle pour aménagements hydroélectriques en cascade ». 6 Conférence Internationale Francophone d'Automatique, Nancy, 2010.

http://hal.archives-ouvertes.fr/inria-00460912/en/

KALMAN R.E., «A new approach to linear filtering and prediction problems ». Transactions of the ASME Journal of Basic Engineering, 82 (1960) : 35-45.

KALMAN R.E., BuCY R.S., "New results in linear filtering and prediction theory ». Transactions of the ASME Journal of Basic Engineering, 83 (1961) : 95-107.

Karin O., Swisa A., Glaser B., Dor Y., Alon U., «Dynamical compensation in physiological circuits ». Molecular Systems Biology, 12 (2016) : 886. DOI 10.15252 /msb. 20167216

QI X., LI J., XIA Y., GAO Z., «On the robust stability of active disturbance rejection control for SISO systems ». Circuits, Systems, and Signal Processing, 36 (2017) : 65-81.

LAFONT F., BALMAT J.-F., PESSEL N., FLIESS M., «A model-free control strategy for an experimental greenhouse with an application to fault accommodation ». Computers and Electronics in Agriculture, 110 (2015) : 139-149.

LAMnABHI-LAgarrigue F., Analyse des systèmes non linéaires, Paris : Hermès, 1994.

DE LARMinat Ph., Automatique appliquée ( $2^{e}$ éd.). Paris : Hermès-Lavoisier, 2009.

LÉVINE J., Analysis and Control of Nonlinear Systems: A Flatness-based Approach. Berlin : Springer, 2009.

LI D., DING P., GAO Z., « Fractional active disturbance rejection control ». ISA Transactions, 62 (2016) : 109-119.

MADOŃSKI R., HERMAN P., « Model-free control or active disturbance rejection control? On different approaches for attenuating the perturbation ». 20th Mediterranean Conference on Control \& Automation, Barcelone, 2012.

MADOŃSKI R., HERMAN P., "Survey on methods of increasing the efficiency of extended state disturbance observers ». ISA Transactions, 56 (2015) : 18-27.

Mboup M., JoIN C., FLIESS M., « Numerical differentiation with annihilators in noisy environment ». Numerical Algorithms, 50 (2009) : 439-467.

Menhour L., d'Andréa-Novel B., Fliess M., Gruyer D., Mounier H., «An efficient modelfree setting for longitudinal and lateral vehicle control. Validation through the interconnected proSiVIC/RTMaps prototyping platform ». IEEE Transactions on Intelligent Transportation Systems, 2017. DOI: 10.1109/TITS.2017.2699283

Noura H., Theilliol D., Ponsart J.-C., Chamseddine A., Fault-tolerant Control Systems - Design and Practical Applications. Londres : Springer, 2009.

O'DWYER A., Handbook of PI and PID Controller Tuning Rules (3rd ed.). Londres : Imperial College Press, 2009. 
PolónI T., Kolmanovsky I., RohA'-ILkIV B., «Simple input disturbance observer-based control: Case studies ». Journal of Dynamic Systems, Measurement, and Control, 140 (2018) : doi: :10.1115/1.4037298

PONCELET J.-V., Introduction à la mécanique industrielle (3e éd.). Paris : Gauthier-Villars, 1870.

Ramírez-Neria M., Sira-Ramírez H., Garrido-Moctezuma R., LuViano-JuÁrez A., "Linear active disturbance rejection control of underactuated systems: The case of the Furuta pendulum ». ISA Transactions, 53 (2014) : 920-928.

Rigatos G.G., Nonlinear Control and Filtering Using Differential Flatness Approaches - Applications to Electromechanical Systems. Heidelberg : Springer, 2015.

RISLER J.-J., Méthodes mathématiques pour la CAO. Paris : Dunod, 1991. Traduction anglaise : Mathematical Methods in CAD. Cambridge : Cambridge University Press, 1992.

ROTELLA F., ZAMBETTAKIS I., «Commande des systèmes par platitude ». Techniques de l'Ingénieur S7450 V1, 2007.

Rudin W., Functional Analysis. New York: McGraw-Hill, 1967. Traduction française : Analyse fonctionnelle, Paris : Ediscience, 2000.

Rudin W., Real and Complex Analysis. New York: McGraw-Hill, 1976. Traduction française : Analyse réelle et complexe, Paris : Dunod, 2009.

RUDOLPH J., Beiträge zur flachheitsbasierten Folgeregelung linearer und nichtlinearer Systeme endlicher und unendlicher Dimension. Aachen : Shaker Verlag, 2003.

RuGH W.J., Nonlinear System Theory - The Volterra/Wiener approach. The Johns Hopkins University Press, Baltimore, 1981.

Sira-RamíRez H., Agrawal S.K., Differentially Flat Systems. New York : Marcel Decker, 2004.

Sira-Ramírez H., García-Rodríguez C., Cortès-Romero J., Luviano-JuÁrez A., Algebraic Identification and Estimation Methods in Feedback Control Systems. Chichester : Wiley, 2014.

Sira-Ramírez H., LuVIANO-JuÁREZ A., Cortès-Romero J., "Control lineal robusto de sistemas no lineales diferencialmente planos ». Revista Iberoamericana de Automática e Informática Industrial, 8 (2011) : 14-28.

Sira-Ramírez H., LuViano-JuÁrez A., Ramírez-Neria M., Zurita-Bustamante E.W., Active Disturbance Rejection Control of Dynamic Systems: A Flatness Based Approach. Oxford \& Cambridge, MA : Elsevier, 2017.

SiRA-RAmíREZ H., ZURITA-BUStAMANTE E.W., LUVIANO-JUÁREZ A., « Robust flat filtering control of a nonlinear manipulator-direct current motor system ». Journal of Dynamic Systems Measurement and Control, 140 (2018) : doi:10.1115/1.4037386

SUN L., DONG J., LI D., LEE K.Y., « A practical multivariable control approach based on inverted decoupling and decentralized active disturbance rejection control ». Industrial \& Engineering Chemistry Research, 55 (2016) : 2008-2019.

TAVASOLI A., ENJILELA V., «Active disturbance rejection and Lyapunov redesign approaches for robust boundary control of plate vibration ». International Journal of Systems Sciences, 48 (2017) : 1656-1670.

ThaBet H., AYADI M., Rotella F., « Design of adaptive PID controllers based on adaptive Smith predictor for ultra-local model control ». International Journal of Automation and Control, 11 (2017) : 222-238.

TICHERFATINE M., QIDAM Z. (2017). «Model-free approach based intelligent PD controller for vertical motion reduction in fast ferries ». Turkish Journal of Electrical Engineering \& Computer Sciences, 2017 DOI: $10.3906 / e l k-1703-224$

Vincent J., Morris D., Usher N., Gao Z., Zhao S., Nicoletti A., Zheng Q., « On active disturbance rejection based control design for superconducting RF cavities ". Nuclear Instruments and Methods in Physics Research A, 643 (2011) : 11-16.

WU Y.-J., HUA Y.Y., LIU X.-D., « Design method of active disturbance rejection variable structure control system ». Mathematical Problems in Engineering, 2015. http:/ / dx. doi .org/10 .1155/2015/195720

XIA Y., Fu M., SHI P., Analysis and Synthesis of Dynamical Systems with Time-Delays. Lecture Notes in Control and Information Sciences 387, Berlin : Springer, 2009.

YAO J., DENG W., "Active disturbance rejection adaptive control of uncertain nonlinear systems: theory and application ». Nonlinear Dynamics, 89 (2017) : 1611-1624.

WANG H., TIAN Y., HAN S., WANG X., "ZMP theory-based gait planning and model-free trajectory tracking control of lower limb carrying exoskeleton system ». Studies in Informatics and Control, 26, 161-170, 2017. 
WANG H., Ye X., TIAN Y., Zheng G., Christov N., « Model-free based terminal SMC of quadrotor attitude and position ». IEEE Transactions on Aerospace and Electronic Systems, 52, 2519-2528, 2016.

ZHANG Y., FAN C., ZHAO F., AI Z., GONG Z., «Parameter tuning of ADRC and its application based on CCCSA ». Nonlinear Dynamics, 76 (2014) : 1185-1194.

Zhang X., Wang H., Tian Y., Peyrodie L., Wang X., " Model-free based neural network control with timedelay estimation for lower extremity exoskeleton ». Neurocomputing, 272 (2018) : 178-188.

ZHAO S., GAO Z., « Modified active disturbance rejection control for time-delay systems ». ISA Transactions, 53 (2014) : 882-888.

ZHENG Q., GAO Z., " Active disturbance rejection control: between the formulation in time and the understanding in frequency ». Control Theory and Technology, 14 (2016) : 250-259.

ZIEGLER J.G., NICHOLS, N.B. (1942). «Optimum settings for automatic controllers ». Transactions of the ASME, 64 (1942) : 759-768.

ZIEGLER J.G., NICHOLS, N.B. (1943). «Process lags in automatic control circuits ». Transactions of the ASME, 65 (1943) : 433-444. 